\title{
Emodialisi a oltranza o dialisi peritoneale?
}

\author{
G. D’Adamo ${ }^{1}$, A. Scipioni ${ }^{2}$, G. Pasquini ${ }^{3}$, L. Lui ${ }^{1}$, F. Forte ${ }^{1}$, C. Spinelli ${ }^{1}$ \\ UUOOCC di ${ }^{1}$ Nefrologia e Dialisi \\ ${ }^{2}$ Radiologia Ospedaliera \\ ${ }^{3}$ Chirurgia Generale, Ospedale S. Spirito - ASL Roma E
}

Nel mese di agosto 2008, una paziente di 43 anni ci viene inviata per esaurimento degli accessi vascolari. Di rilievo nell'anamnesi remota un intervento di cistectomia e ureterosigmoidostomia per cistite interstiziale eseguito all'età di 14 anni. All'età di 16 anni era poi stata diagnosticata idroureteronefrosi dx ed era stata collocata e mantenuta in sede per tre anni una derivazione pielostomica. Successivamente la paziente non aveva più effettuato controlli clinici e di laboratorio fino al dicembre 2003, quando era stata ricoverata a seguito di vomito incoercibile e sottoposta ad emodialisi per la presenza di insufficienza renale e grave acidosi metabolica.Il trattamento, iniziato in urgenza da catetere in vena femorale $\mathrm{dx}$, era poi proseguito ambulatorialmente sempre utilizzando cateteri vascolari per l'accesso dialitico.

All'ingresso nel nostro ospedale, la paziente presentava astenia marcata, nausea e vomito, peso corporeo $54.5 \mathrm{~kg}$ (+2 kg rispetto al peso forma), creatininemia $21.3 \mathrm{mg} /$ $\mathrm{dL}$, azotemia $230 \mathrm{mg} / \mathrm{dL}$, potassiemia $6.6 \mathrm{mmol} / \mathrm{L}, \mathrm{Hb}$ $6.2 \mathrm{~g} / \mathrm{dL}, \mathrm{pH} 7.13, \mathrm{HCO}_{3}-13 \mathrm{mmol} / \mathrm{L}$. Erano in sede e non funzionanti un catetere, recentemente collocato con tecnica chirurgica in vena giugulare interna sinistra, e protesi vascolari rette tra arteria omerale e vena ascellare, ad entrambi gli arti superiori. In regione laterocervicale dx e sin esiti cicatriziali di pregressi cateterismi delle vene giugulari. All'inguine, bilateralmente, esiti infetti di pregresse collocazioni chirurgiche in vena femorale di cateteri per emodialisi.

Per effettuare un'indagine flebografica, il radiologo vascolare tentava senza successo la puntura della vena giugulare destra. L'iniezione di m.d.c. nel catetere posizionato in vena giugulare interna sinistra dimostrava obliterazione delle vene giugulari, salvo il terzo superiore della giugulare interna destra, e obliterazione delle vene succlavie e della vena cava superiore (Fig. 1). La successiva iniezione di m.d.c. attraverso le vene femorali dimostrava la pervietà delle vene femorali comuni, con scarico endopelvico attraverso collaterali otturato- rie e del plesso ovario, obliterazione delle vene iliache esterne, vena ovarica destra dilatata (Figg. 2 e 3 ).

Risultava pertanto impossibile procedere alla collocazione di un ulteriore catetere vascolare nelle sedi usuali. Il contatto con i Centri nazionali con esperienza di accessi vascolari "estremi" non aveva reso possibile il trasferimento immediato della paziente per tentare la collocazione translombare o chirurgica di un catetere per emodialisi in vena cava inferiore (1).

$\mathrm{Si}$ è quindi deciso di tentare la collocazione di un catetere peritoneale, nonostante la storia di pregresso intervento chirurgico addominale maggiore. Il posizionamento con tecnica chirurgica del catetere peritoneale è stato effettuato senza difficoltà e la dialisi peritoneale è stata iniziata immediatamente, erogando piccoli volumi di soluzione dialitica con tecnica automatizzata, a paziente supina, per ridurre il rischio di leakage precoce (2). Nonostante le precauzioni adottate, in sesta giornata si è manifestato leakage di soluzione dialitica dal foro di uscita del catetere e si è reso necessario un ulteriore intervento di revisione del catetere per realizzare un migliore ancoraggio della cuffia profonda con collante acrilico. Dopo un ulteriore periodo di due settimane di APD a bassi volumi, la paziente è stata istruita all'esecuzione della CAPD.

Da gennaio 2009, dopo 5 mesi di CAPD, si è osservata la ripresa di regolari cicli mestruali e, a 10 mesi dallinizio della DP, clearance della creatinina (52 L/sett/1.73mq), $\mathrm{KT} / \mathrm{V}(1.8)$ e ultrafiltrazione $(900 \mathrm{~mL} / \mathrm{die})$ sono adeguati.

\section{Discussione}

Il caso descritto offre lo spunto ad alcune considerazioni sull'utilizzo della dialisi peritoneale.

In primo luogo, senza nulla togliere all'efficacia della dialisi peritoneale quale trattamento "di salvataggio" per una paziente con esaurimento degli accessi vascolari, 


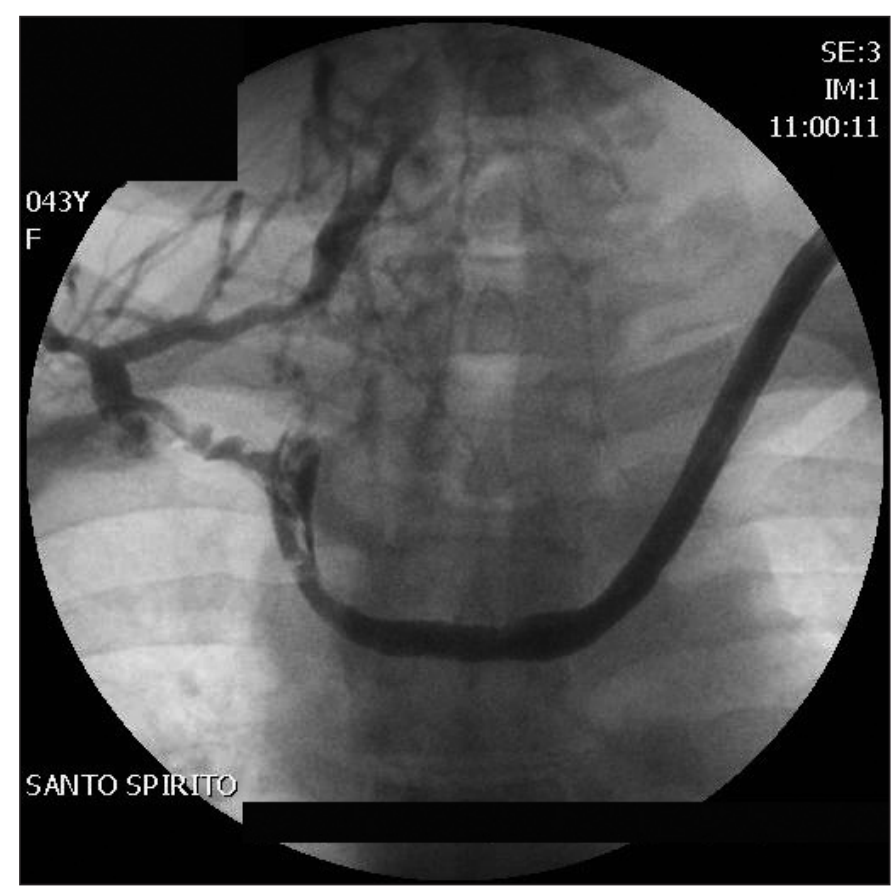

Fig. 1 - Attraverso il catetere precedentemente posizionato sono stati valutati il circolo venoso giugulare e il tronco anonimo con mancata visualizzazione del circolo giugulare superficiale, del tronco anonimo e della vena cava superiore in accordo con trombosi venosa diffusa.

si deve tuttavia notare che sarebbe stato più opportuno indirizzarla alla DP all'inizio del trattamento o almeno dopo il fallimento dei primi accessi vascolari. Il migliore utilizzo della DP è infatti all'inizio di un programma integrato di gestione dell'uremia, in presenza di funzione renale residua, mentre si è in attesa del trapianto (3). È dimostrato che il ricorso alla DP all'inizio della terapia sostitutiva preserva più a lungo la funzione renale residua (4) e consente di risparmiare il patrimonio vascolare per eventuali future necessità emodialitiche (3). La paziente non era invece a conoscenza dell'esistenza della dialisi peritoneale fino a quando non ci è stata inviata. Va detto che probabilmente la DP non sarebbe stata comunque consigliata come primo trattamento in considerazione del precedente intervento addominale maggiore. Inoltre la paziente non avrebbe probabilmente scelto la DP come primo trattamento, perché non particolarmente orientata all'autocura, anche se si è poi dimostrata perfettamente in grado di eseguire le manovre dialitiche e la medicazione dell'emergenza cutanea del catetere.

I ripetuti cateterismi vascolari effettuati per proseguire il trattamento con l'emodialisi, nonostante il fallimento di tutti i tentativi di confezionare una fistola naturale o protesica, potrebbero verosimilmente aver contribuito a

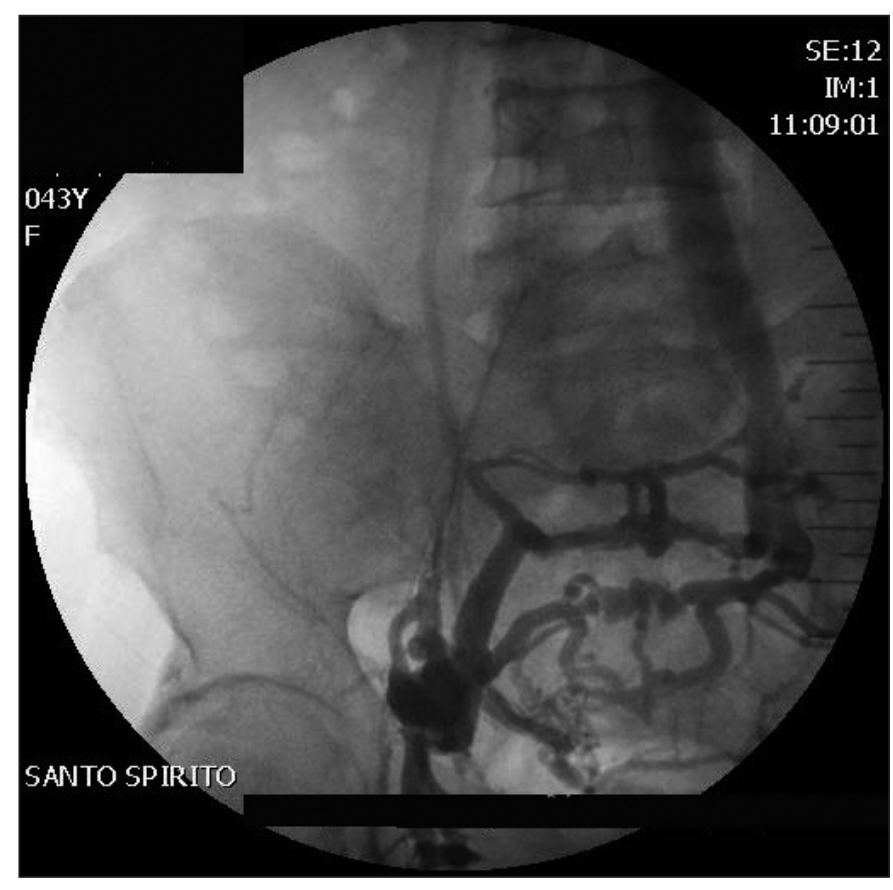

Fig. 2 - La puntura diretta della femorale di destra documenta trombosi dell'asse iliaco-femorale con evidenza di doppia cava che si opacizza tramite la vena iliaca interna ed i circoli profondi pelvici. In particolare, appaiono ben documentate la vena cava di sinistra lungo tutto il suo decorso, la morfologia filiforme della cava di destra e la vena ovarica destra.

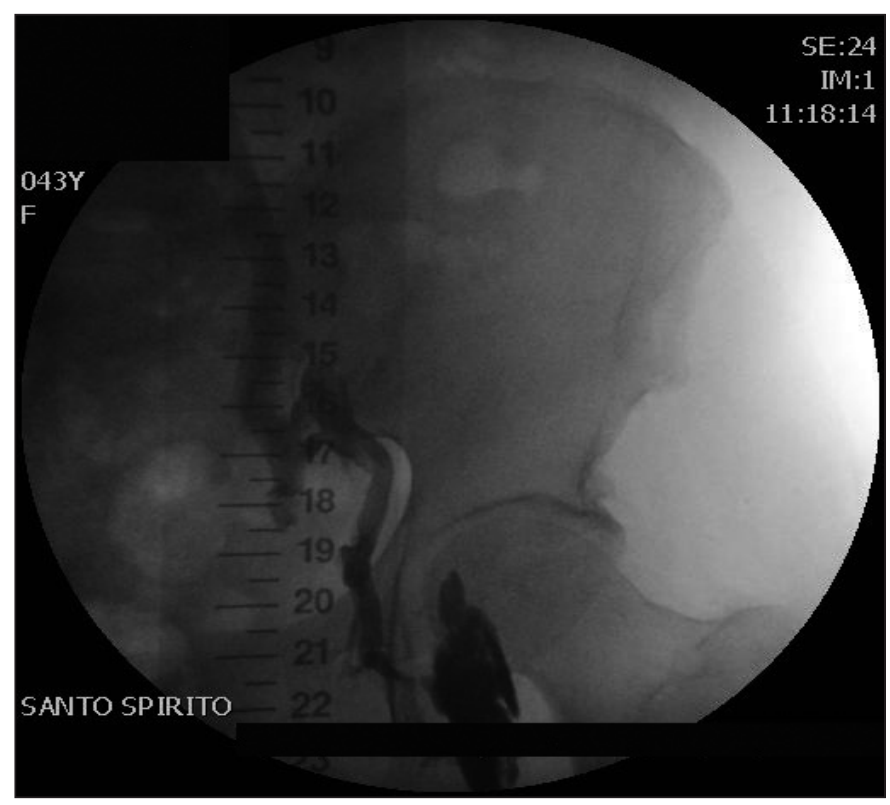

Fig. 3 - La puntura diretta della vena femorale sinistra documenta pervietà della vena iliaca interna sinistra che confluisce nella vena cava omolaterale descritta nella Figura precedente.

Non documentabili i vasi pelvici profondi e la vena iliaca esterna sinistra. 
determinare le trombosi venose multiple che sono state dimostrate (5). Non risulta infatti che siano state diagnosticate in passato delle alterazioni della coagulazione $\mathrm{e}$ l'attuale situazione vascolare della paziente sconsiglia di interrompere la somministrazione di eparina a basso peso molecolare per eseguire lo screening per la trombofilia. Il patrimonio vascolare residuo è ormai talmente esiguo da rendere al momento impossibile un programma di trapianto renale e questo ci porta all'ultima, ma non meno importante questione: per quanti anni sarà possibile proseguire la dialisi peritoneale? Non molti pazienti hanno effettuato con beneficio la DP per più di 10 anni (6) e oggi sappiamo che il rischio di sviluppare peritonite sclerosante aumenta con l'aumentare dell'età dialitica (7).

Il problema della nostra paziente può dunque considerarsi risolto solo temporaneamente.

\section{Indirizzo degli Autori:}

Gianna D'Adamo, MD

UOC Nefrologia e Dialisi

Ospedale S. Spirito

ASL Roma/E

giannadadamo@tiscali.it

\section{Bibliografia}

1. Gupta A, Karak PK and Saddekni S. Translumbar inferior vena cava catheter for long-term hemodialysis. JASN 1995; 5 (12): 2094-7.

2. Linee Guida per la dialisi peritoneale (DP). G Ital Nefrol 2003; 20 (S-24): S109-S28.

3. Lameire N, Van Biesen W, Vanholder R. The role of peritoneal dialysis as first modality in an integrative approach to patients with end-stage renal disease. Perit Dial Int 2000; 20 (Suppl 2): S134-41.

4. Hidaka $\mathrm{H}, \mathrm{Nakao}$ T. Preservation of residual function and factors affecting its decline in patients on peritoneal dialysis. Nephrology (Carleton) 2003; 8: 184-91.
5. Bander SJ, Schwab SJ. Central venous angioaccess for hemodialysis and its complications. Semin Dial 1992; 5: 121-8.

6. Gokal R. Long term peritoneal dialysis-is it a reality? J Nephrol 1999; 12(6): 362-70.

7. D’Adamo G. La peritonite sclerosante in breve. Giornale di Tecniche Nefrologiche \& Dialitiche 2007; 19(2): 16-8.

\section{TEST di VERIFICA - 4}

1) Quale delle seguenti è controindicazione asso luta alla dialisi peritoneale?

a) Malattia renale policistica

b) Impossibilità di inserire il catetere peritoneale

c) Ernia inguinale

d) Età avanzata

2) In un programma integrato di gestione dell'uremia la dialisi peritoneale va effettuata

a) Preferibilmente dopo l'esaurimento degli accessi vascolari per emodialisi

b) Preferibilmente all'inizio del trattamento sostitutivo

c) In attesa della "maturazione" della fistola artero-venosa per emodialisi

d) Solo nei bambini

3) Per la prevenzione del leakage (stravaso di soluzione dialitica) precoce è utile iniziare la DP

a) Con volumi ridotti di soluzione dialitica

b) Dopo un periodo di "break in" di 2-3 settimane

c) Mantenendo il paziente in posizione supina

d) Tutte le precedenti

e) Nessuna delle precedenti

Le risposte corrette alle domande sono a pag. 53 